\title{
Erratum to: Candidatus Brocadia and Candidatus Kuenenia predominated in anammox bacterial community in selected Chinese paddy soils
}

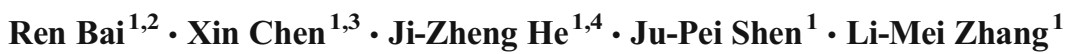

Published online: 14 August 2015

(C) Springer-Verlag Berlin Heidelberg 2015

Erratum to: J Soils Sediments

DOI 10.1007/s11368-015-1131-5

An error occurred in one of the foundation code numbers in the Acknowledgments.

Incorrect version:

Acknowledgments This work was financially supported by the National Science Foundation of China (41090281, 41025004, 41322007). We would like to thank Prof. Wenxue Wei and Ms. Yijun Zhu for their assistance in soil sampling and Drs. Peng Cao and Yu Dai for their help in soil physiochemical analysis.

Correct version:

Acknowledgments This work was financially supported by the National Science Foundation of China (41090281, 41203053, 41322007). We would like to thank Prof. Wenxue Wei and Ms. Yijun Zhu for their assistance in soil sampling and Drs. Peng Cao and Yu Dai for their help in soil physiochemical analysis.

The online version of the original article can be found at http://dx.doi.org/ 10.1007/s11368-015-1131-5.

Li-Mei Zhang

zhanglm@rcees.ac.cn

1 State Key Laboratory of Urban and Regional Ecology, Research Centre for Eco-Environmental Sciences, Chinese Academy of Sciences, 100085 Beijing, China

2 University of Chinese Academy of Sciences, 100049 Beijing, China

3 State Key Laboratory of Atmospheric Boundary Layer Physics and Atmospheric Chemistry, Institute of Atmospheric Physics, Chinese Academy of Sciences, 100029 Beijing, China

4 Melbourne School of Land and Environment, The University of Melbourne, Parkville 3010, Victoria, Australia 